\section{Innovations in Policy and Practice: A work in progress}

In this edition of the Journal, we publish our first two articles in the new section on Innovations in Policy and Practice (IPP). We launched the section in January 2016, and within the first six months have received 28 submissions, which is a promising start. In this editorial, I provide an update on how we dealt with these, which will give potential authors some guidance for their own submissions; review the lessons we have learned and the changes we have made as a result; and discuss future directions.

There are several innovations in the IPP section that distinguish it from the rest of the journal. The first of these is that we require authors to first submit a 250-word abstract; initially, this was a 100 -word synopsis, but we found that this was insufficient to make a judgement, creating some delays as we sought clarification, so that was recently changed. The abstracts are reviewed by me and one or two of the Associate Editors (Connie Clement, Cory Neudorf, Jane Underwood, Marie-France Raynault, and Richard Massé), all of whom are well-known practitionerscholars. We may seek clarification from the author on certain points or suggest areas of the proposed article we see as particularly interesting and worth focusing on.

Of the first 28 submissions, we have: recommended that 12 be submitted to the journal as full articles, turned down 11, suggested one be submitted to the journal as a regular Commentary, and requested further details from four; for some of these, it has been a couple of months or more since the decision was sent, so they may in fact no longer be active. Of the 12 we recommended for fullarticle submission, six have been submitted and are at various stages of the reviewing process; the first two, of course, are published in this edition.

For most of the 12 recommended for submission, we have usually made suggestions of areas to focus on. We have also made clear that a recommendation for submission is not the same as acceptance. Once the article is submitted, through the usual online process, it undergoes peer review, and subsequently may be rejected, sent back for revision or accepted.

However, the peer review process is different in two respects; all the reviewers are truly peers, in that they are practitioners first and foremost, but with experience in publication and research. Second, the reviewing process is non-blind; authors and reviewers are identified to each other. Our hope is that the review process will be more collegial, and may even become a form of mentorship or coaching. However, as we have only had five go through the review process, it is still too early to judge whether this will work the way we hope it will.

There were three main reasons why we turned down 11 at initial review; five were innovative model proposals or ideas that had not been implemented, four were not public health topics (two were primary care, one was hospital services, one was about coaching/ teaching students) and two were neither policy nor practice but were epidemiological studies or surveys.

This has led us to be more explicit in our revised author guidelines that we are interested in innovations that have been implemented, not simply in innovative ideas, models and suggestions. While the latter may well be useful, we believe that

\section{Innovations dans les politiques et la pratique : un travail en chantier}

Dans ce numéro de la Revue, nous publions les deux premiers articles de notre nouvelle rubrique sur les Innovations dans les politiques et la pratique (IPP). Nous avons créé cette rubrique en janvier 2016, et en six mois à peine, nous avons reçu 28 manuscrits... un début prometteur. Dans les paragraphes suivants, j'explique comment nous avons traité ces envois afin de donner aux auteurs potentiels quelques principes directeurs pour leurs propres manuscrits; je présente les leçons que nous avons apprises et les modifications que nous avons apportées; et je réfléchis à nos orientations futures.

Plusieurs innovations de la rubrique des IPP la distinguent du reste de la Revue. La première est que nous demandons aux auteurs de soumettre d'abord un résumé en 250 mots; au départ, nous demandions un sommaire en 100 mots, mais nous avons constaté que c'était insuffisant pour porter un jugement et que cela causait des retards lorsqu'il fallait demander des éclaircissements; nous avons donc modifié ce critère récemment. Les résumés sont évalués par moi et par un ou deux rédacteurs scientifiques adjoints (Connie Clement, Cory Neudorf, Jane Underwood, Marie-France Raynault et Richard Massé), tous des praticiensuniversitaires connus. Il nous arrive de demander des éclaircissements aux auteurs sur certains points ou de souligner les aspects de l'article proposé que nous trouvons particulièrement intéressants et qu'il serait bon de privilégier.

Sur les 28 premiers manuscrits reçus, nous avons : recommandé dans 12 cas que l'article au long soit soumis à la Revue, refusé 11 manuscrits, suggéré qu'un manuscrit soit soumis en tant que Commentaire ordinaire, et demandé des précisions au sujet des quatre autres; dans certains cas, il s'est écoulé deux mois ou plus depuis que la décision a été rendue, et les manuscrits en question pourraient ne plus être actifs. Sur les 12 pour lesquels nous avons recommandé la soumission de l'article complet, six ont été soumis et en sont à diverses étapes du processus d'évaluation; les deux premiers sont évidemment publiés dans ce numéro.

Pour la plupart des 12 résumés pour lesquels nous avons recommandé la soumission de l'article complet, nous avons en général suggéré des aspects à privilégier. Nous avons aussi précisé qu'une telle recommandation ne signifie pas que l'article sera accepté. Une fois l'article soumis par les voies habituelles (en ligne), il fait l'objet d'une évaluation par les pairs, après quoi il est soit refusé, soit retourné pour révision, soit accepté.

Le processus d'évaluation par les pairs pour la rubrique IPP est différent à deux égards; tous les évaluateurs sont réellement des pairs, à savoir que ce sont d'abord et avant tout des praticiens, mais qui ont de l'expérience en publication et en recherche. Deuxièmement, l'évaluation ne se fait pas en aveugle; les auteurs connaissent l'identité des évaluateurs et vice versa. Nous espérons qu'ainsi le processus d'évaluation sera plus collégial et qu'il deviendra même une forme de mentorat ou d'encadrement. Comme il n'y a eu que cinq manuscrits évalués jusqu'à maintenant, il est encore trop tôt pour voir si cela fonctionnera comme nous l'espérons.

Il y a trois grandes raisons pour lesquelles nous avons refusé 11 manuscrits dès l'examen initial : cinq proposaient des idées ou des modèles novateurs qui n'avaient pas été mis en œuvre, quatre ne portaient pas sur la santé publique (deux sur les soins primaires, un sur les services hospitaliers et un autre sur l'enseignement/l'encadrement des étudiants) et deux ne concernaient ni les politiques, ni la pratique, mais des études épidémiologiques ou des enquêtes.

Cela nous a amenés à préciser, en révisant nos lignes directrices aux auteurs, que nous nous intéressons aux innovations qui ont été 
what matters to practitioners is practice: This has been tried, we could learn from it, adapt it if need be, and apply it where we work.

And what of the two we are publishing here? One is in itself innovative in that it is a short report of 1,000 words, a format we think may be very appropriate for practitioners, especially for reporting early results of an innovation that seems promising but needs further dissemination and evaluation. It describes an interesting approach to bringing public health and other services to people who are homeless through free pet care services provided by veterinary staff. By establishing a human contact and a sense of trust, it may be possible to draw homeless people into accepting public health services that they need but have not wished to or been able to access.

The second article is particularly timely, given the epidemic of opioid overdose deaths in Canada. While this was a process evaluation and not an outcome evaluation, it does provide an early report on a program in Toronto that provides opioid overdose education and distribution of naloxone, and seems to perform quite well.

Other articles in the pipeline include the implementation of healthy eating and active living guidelines in municipal child care settings, which engaged the cooks within the settings and sport and activity coaches from outside the setting; a digital gaming intervention in movie theatres for STI and HIV prevention; an evaluation of improved linkage to HIV care at low-threshold STI/ HIV testing sites; and "pathways to policy" that were identified as critical success factors facilitating and accelerating the development and implementation of physical activity and built environment policy. So as you can see, we have already got a wide variety of innovations, from the clinical to the policy level, which is what we hoped for.

As to the future, we have some further innovations in mind we hope to implement over time. We have not yet got to the point where we have organized in-person discussions between authors and reviewers, but we hope to do so. We also hope to be able to publish Powerpoints or other visuals, and provide links to websites and on-line videos that will complement the articles, although some of those must await the further evolution of our electronic publishing platform.

We also need new reviewers, so if you are an experienced practitioner with a track record of publications and/or research, we would be happy to hear from you and add you to our list.

But ultimately, the success of this new section of the journal depends upon you - the practitioner-readers. As it develops, we want to hear back from you as to whether it meets your needs, how we can improve the section, what else you need. Above all, we want your submissions. So if you are a practitioner and you have an innovative approach you have implemented, submit an abstract on the journal site - we would be happy to review it and let you have our thoughts. We look forward to hearing from you!

Trevor Hancock, Senior Editor, CJPH

Thancock@uvic.ca

doi: $10.17269 /$ CJPH.107.5819 mises en œuvre, et non pas simplement aux idées, aux suggestions ou aux modèles novateurs. Ceux-ci ont leur utilité, mais nous croyons que ce qui compte pour les praticiens, c'est la pratique : nous avons essayé telle chose, nous pouvons en tirer des leçons, l'adapter au besoin et l'appliquer dans notre milieu de travail.

Qu'en est-il des deux articles publiés dans ce numéro? L'un est novateur en soi, car c'est un bref rapport de 1000 mots, une formule qui pourrait être très pertinente pour les praticiens selon nous, surtout pour faire connaître les premiers résultats d'une innovation qui semble prometteuse, mais qui n'a pas encore été diffusée ni évaluée. L'article décrit une façon intéressante de mettre des services de santé publique (ou autres) à la portée des personnes sans abri en proposant des services de soins aux animaux de compagnie donnés gratuitement par du personnel vétérinaire. Le contact humain et la mise en confiance pourraient être des moyens de convaincre les sansabri d'accepter les services de santé publique dont ils ont besoin, mais auxquels ils ne souhaitent pas ou ne peuvent pas avoir accès.

Le second article est particulièrement à propos, vu l'épidémie de décès par surdose d'opioïdes qui sévit au Canada. Il porte sur une évaluation des processus et non des résultats, mais donne un premier aperçu d'un programme torontois qui offre de la formation permettant d'intervenir avec de la naloxone en cas de surdoses d'opioïdes; ce programme semble très bien fonctionner.

Les autres articles en préparation portent : sur l'application de lignes directrices sur l'alimentation saine et la vie active dans des services de garde de responsabilité municipale, où l'on a impliqué, à l'interne, les cuisiniers membres du personnel et recruté des entraîneurs sportifs et coordonnateurs d'activités de l'extérieur; sur une intervention de prévention des ITS et du VIH par un jeu numérique projeté dans les cinémas; sur l'évaluation d'un lien amélioré vers les soins du VIH dans les centres de dépistage des ITS/du VIH à seuil bas; et sur les «sentiers stratégiques » définis comme étant des facteurs de réussite déterminants pour faciliter et accélérer l'élaboration et la mise en œuvre de politiques sur l'activité physique et l'environnement bâti. Comme nous l'espérions, nous avons donc déjà des innovations très diverses, du niveau clinique à celui des politiques.

Pour l'avenir, nous songeons à d'autres innovations à mettre en œuvre au fil du temps. Nous n'avons pas encore eu le loisir d'organiser des discussions en personne entre auteurs et évaluateurs, mais nous espérons le faire. Nous aimerions aussi pouvoir publier des présentations PowerPoint (ou sur d'autres supports visuels) et fournir des liens vers des sites Web et des vidéos en ligne pour compléter les articles, mais certaines de ces idées devront attendre la modernisation de notre plateforme d'édition électronique.

Nous cherchons également de nouveaux évaluateurs, donc si vous êtes une praticienne ou un praticien d'expérience avec des publications et/ou de la recherche à votre actif, nous attendons de vos nouvelles et nous serons ravis de vous ajouter à notre liste.

Mais en bout de ligne, c'est vous, les praticiens-lecteurs, qui déciderez du succès de la nouvelle rubrique de la Revue. Au fil de son évolution, nous aimerions que vous nous disiez si elle répond à vos besoins, comment nous pourrions l'améliorer et quels sont vos autres besoins. Par-dessus tout, nous voulons vos manuscrits. Donc, si vous êtes praticien-praticienne et que vous avez appliqué une approche novatrice, soumettez un résumé sur le site de la Revue; nous serons heureux de l'examiner et de vous dire ce que nous en pensons. Au plaisir de vous lire!

Trevor Hancock, Rédacteur, RCSP

Thancock@uvic.ca 